\title{
Écrans de soutènement Interaction sol-structure, à propos de la méthode des modules de réaction
}

\section{P. VEZOLE}

Société Auxiliaire

d'Entreprises

143, avenue de Verdun 92442 Issy-les-Moulineaux cedex
La méthode des coefficients de réaction repose sur I'hypothèse de proportionnalité entre les incréments de pression et les incréments de déplacement le long de la surface d'appui d'une structure. Cette méthode ne peut donc pas, sauf cas particulier, conduire à une modélisation réaliste de l'interaction sol-structure, et la crédibilité qui lui est souvent accordée pour les écrans de soutènement est totalement injustifiée.

II est urgent de développer des entrées, sorties, et commandes spécialisées simplifiant l'utilisation de codes d'application de la méthode des éléments finis ou de la méthode des différences finies pour l'étude des projets courants de soutènement, les codes généraux restant disponibles pour l'étude de cas complexes.

Retaining walls

The reaction coefficients method rests on the hypothesis that proportionnality exists between pressure increments and displacement increments along the structure support surface. This method thus cannot, except for specific case, lead to a realistic modelisation of the ground/structure interaction. The credit often attributed to it for the retaining walls is completely injustified.

It is urgent to develop entries, exits and special orders which would simplify the use of the application codes of the finite elements or the finite differences analysis method to design simple retaining walls, the general codes being still available for the more complex cases. 
A force d'utiliser un outil, l'esprit critique s'émousse et, compte tenu des services rendus, on oublie les imperfections de cet outil et on lui prête des qualités qu'il n'a pas.

Le présent article peut être perçu comme étant quelque peu provocateur ou, parfois même, outrancier. Mais la situation nécessite sans doute une prise de position catégorique, car les dérives sont graves :

- Il est fort décevant de constater que des efforts importants sont consentis pour perfectionner la (Méthode des Coefficients de Réaction n), dépourvue de toute base scientifique, alors que des approches beaucoup plus réalistes sont l'objet de beaucoup moins d'attentions.

- Cette méthode est utilisée par des gens peu avertis des subtilités de la mécanique des sols, et qui accordent une confiance aveugle à des logiciels qui sont perçus comme des références ; ce n'est d'ailleurs pas de leur faute " s'ils y croient n, puisque diverses publications récentes, jusque dans la $R F G$, présentent des « raffinements » de la Méthode, lui conférant d'après les auteurs un réalisme satisfaisant...

- La méthode est utilisée sans grande précaution pour les écrans de soutènement, qui nous préoccupent aujourd'hui, mais aussi pour les dallages, les radiers, les réservoirs, les chaussées, les ouvrages sous remblai, les ponts-cadres, etc., et ceci sur la base d'essais dont la représentativité est souvent discutable (l'essai de plaque, pour ne citer que lui, est mis en œuvre dans des conditions souvent scabreuses ; mais, lui non plus, n'est pas l'objet de mises en garde pourtant indispensables !).

La situation me parait assez grave pour que la mise en cause exposée ici soit sans nuance.

\section{Vocabulaire}

Dans le contexte de rigueur de vocabulaire pour la normalisation française ou européenne, il faut signaler qu'il convient de parler de coefficient de réaction et non de module de réaction.

Un abus de langage est sans doute pour quelque chose dans les incompréhensions que l'on constate aujourd'hui entre les gens de structures et les gens de sols : la notion de poutre sur appui continu élastique est confondue avec celle de poutre sur massif élastique.

\section{Évolution des modèles}

Il semblerait que l'évolution des modèles d'interaction sol-structure pour les radiers ait précédé celle concernant les autres ouvrages.

Le premier schéma de calcul est une réaction uniformément répartie (Fig. 1.a).

On perçoit intuitivement que la souplesse relative du radier conduit à une répartition des réactions tout à fait différente, avec sans doute une concentration de contraintes au voisinage immédiat des points d'appui de la structure. Deux schémas sont alors en concurrence : le « faux-radier ») et le « radier sur appui continu élastique $)$.
Dans le cas du faux-radier (Fig. 1.b), l'arbitraire du choix de la répartition des pressions à l'interface est évident, et gênant. Bien des ouvrages, justifiés sur cette base, ont donné et donnent encore satisfaction.

Dans le cas de l'appui continu élastique (Fig. 1.c), l'arbitraire n'est perçu qu'à propos du choix du coefficient de réaction, et il est masqué par de très jolis développements analytiques et la faible sensibilité des résultats à la valeur choisie pour le coefficient. Il est sidérant de constater, lorsqu'on interroge un projecteur de structures, de haut niveau, qu'il est souvent convaincu que la seule erreur apportée par le modèle est l'assimilation du sol à un matériau élastique !

Lorsque les moyens de calcul puissants se sont généralisés, le réflexe a été de se débarrasser des calculs fastidieux induits par la méthode des coefficients de réaction, au lieu de s'efforcer de mettre au point un modèle plus réaliste. Et on en a généralisé I'utilisation pour la modélisation de la plupart des cas d'interaction sol-structure. Et pour faire bon poids, on a rajouté une couche de vernis pseudo-scientifique en qualifiant d'élastoplasticité l'introduction de limites de type coefficient de poussée ou de butée (éléments indispensables pour que le modèle appliqué aux soutènements ne devienne pas trop dangereux en matière de stabilité) : les limites introduites pour les ressorts ne relèvent nullement de l'élastoplasticité, et elles n'ont en pratique pas beaucoup plus de sens physique que les coefficients de réaction!

Ce modèle a pu, dans divers cas, conduire à des prévisions acceptables, ce qui lui confère une crédibilité, que l'on généralise abusivement trop souvent, sans se douter que la crédibilité n'est autre que celle de constructions à l'identique, avec calage du modèle sur

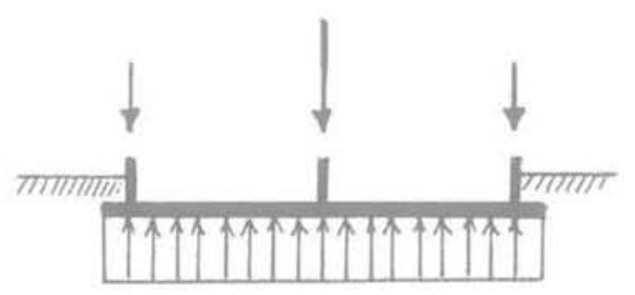

a

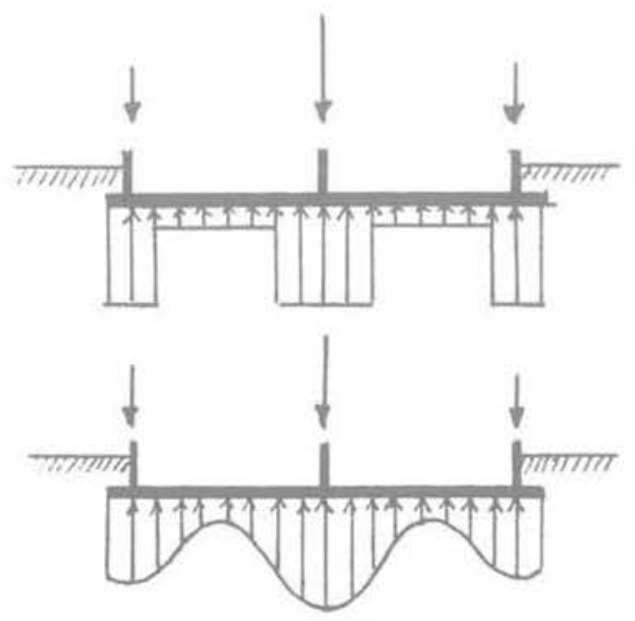

b

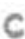


un cas très voisin, et découle aussi de la ductilité du matériau de construction.

La raison principale du fonctionnement satisfaisant des ouvrages dimensionnés par l'une quelconque des approches « simplifiées ») est très probablement l'évolution importante de la répartition de l'interaction qui découle de la moindre amorce de rotule plastique au sein de la structure. Dans le cas, par exemple, d'une semelle de fondation, la méthode des bielles donne satisfaction, en étant basée sur une répartition uniforme de la pression, et on ne peut retrouver des répartitions de pression globalement comparables qu'en tenant compte d'un phénomène d'adaptation correspondant à une fissuration modeste. Plus que la représentativité de la répartition des pressions, la condition du succès en matière de résistance est la ductilité de la structure. Mais cette condition n'est pas toujours suffisante.

Il n'est pas sans intérêt de relire le manuel Mécanique des sols appliquée, de Terzaghi et Peck, à propos du modèle objet des présentes critiques. On rencontre d'abord des mises en garde sans nuance :

"Les procédés simplifiés reposent sur une hypothèse arbitraire et inexacte, à savoir que le tassement $S$ d'un élément quelconque de la surface chargée est absolument indépendant de la charge sur les éléments voisins. On suppose également, ce qui est contraire à la réalité, que le rapport $K_{\mathrm{s}}=p / S$ entre intensité $p$ de la pression sur l'élément et le tassement correspondant $S$ est une constante $K_{s}$,

" Le coefficient $K_{\mathrm{s}}$ a reçu le nom de coefficient de réaction du sol de fondation. y.

Les mises en garde se répètent sur l'ensemble des développements portant sur la méthode des coefficients de réaction, mais on relève néanmoins une phrase " imprudente » si on l'extrait de son contexte (citation tronquée) :

"Étant donné que les calculs sont toujours compliqués, cette étude ne se justifie que si elle conduit à des économies considérables dans le coût de la construction. $n$

\section{2}

\section{Qu'est-ce qu'un coefficient de réaction?}

On sait définir cette notion globalement pour une semelle rigide : $\mathrm{K}$ est le rapport $\mathrm{F} / \delta$ dans un domaine de sollicitation où cette valeur est constante.

C'est une loi mathématique utilisée en Résistance des Matériaux, l'appui continu élastique ; notons que l'obtention physique d'une telle loi est fort malaisée, puisque même les spécialistes que sont les fabricants de matelas à ressorts parviennent mal à éviter que les mouvements d'un dormeur ne soient ressentis par la personne qui partage sa couche. Elle n'a de sens que dans des cas très particuliers comme les jupes de réservoirs cylindriques.

C'est un modèle admis inconditionnellement par beaucoup dans la profession, une référence souvent jugée représentative en matière d'efforts comme de déplacements, pour l'interaction sol-structure.

\section{Cas des éléments linéaires}

Certains professionnels expérimentés accordent une crédibilité certaine à la méthode des coefficients de réaction pour des éléments linéaires tels que les pieux, alors qu'ils restent très réservés vis-à-vis des éléments surfaciques comme les radiers ou les écrans de soutènement.

Sur la figure 2 sont représentés les effets de l'application d'une pression uniforme sur un élément de discrétisation d'une plaque ou d'un ruban. Il apparaît que
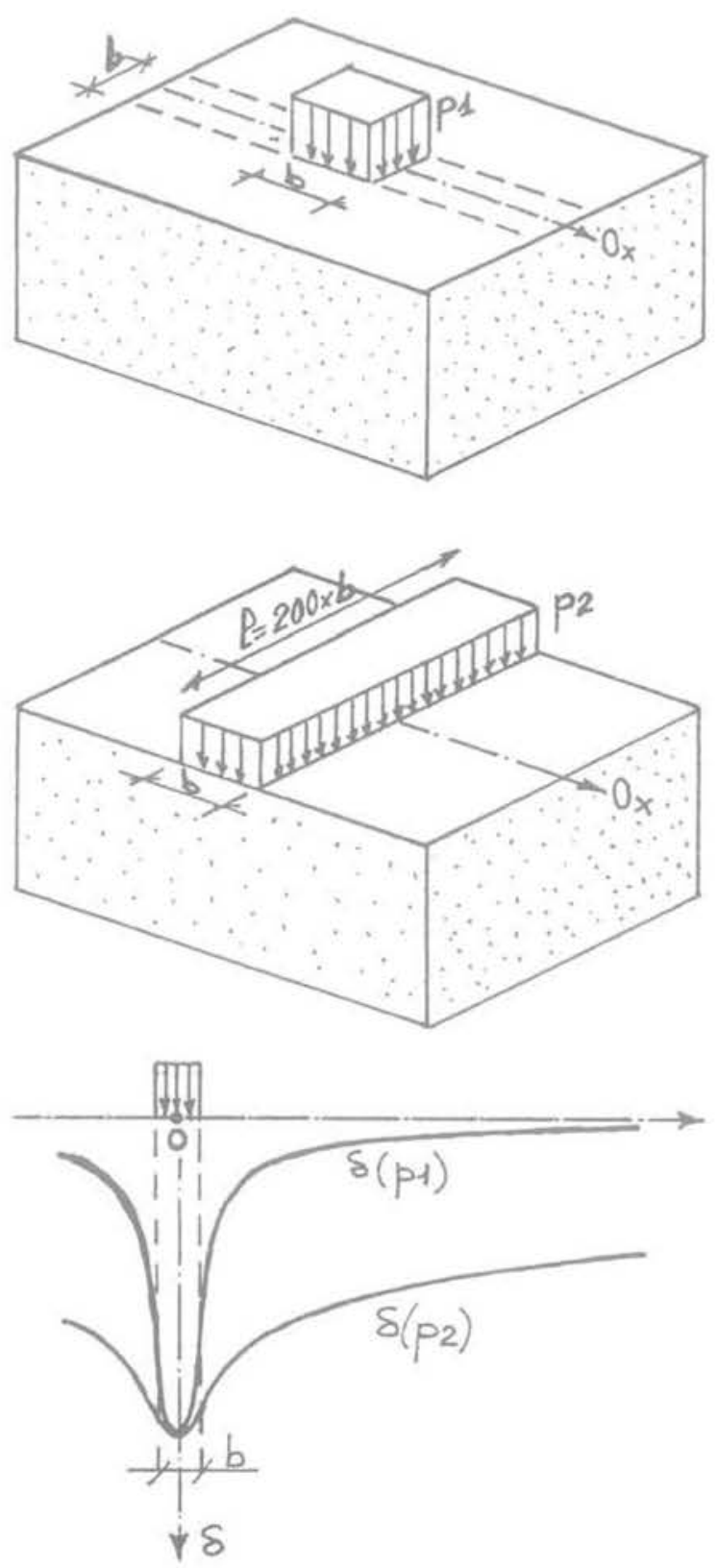

FG. 2. Massif semi-infini élastique, tassements dus à un élément de ruban ou à un élément de plaque. Semi infinite elastic footing, settlements due to a strip element or a plate element. 
le « couplage des ressorts », non pris en compte dans le modèle des coefficients de réaction, est beaucoup plus important dans le cas de la plaque. La méthode n'est pas pour autant rigoureuse dans le cas d'un ruban, bien entendu, mais on perçoit intuitivement que les erreurs n'excèdent sans doute pas l'ordre de grandeur de celles commises sur la détermination des caractéristiques mécaniques du sol.

\section{4}

\section{Calcul inverse de calage sur l'élasticité linéaire}

Comme cela a été dit ci-dessus, de nombreux projeteurs sont convaincus que la seule imperfection du modèle des coefficients de réaction est l'assimilation du sol à un matériau élastique. Plaçons-nous donc dans ce cas d'interaction entre une structure chargée et un
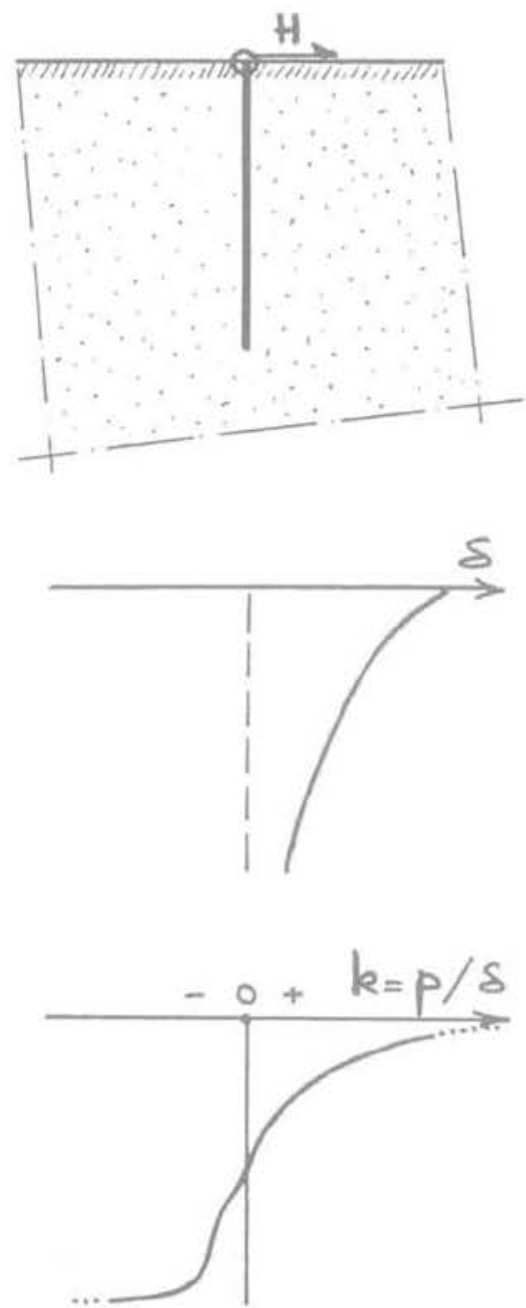

Massif semi-infini élastique, rideau fiché dans le massif, distribution du coefficient de réaction équivalent.

Semi infinite elastic footing, wall restrained in the footing, equivalent reaction coefficient diagram. massif semi-infini constitué d'un matériau au comportement élastique linéaire.

On peut alors calculer les pressions à l'interface et les déplacements dus au chargement. Donc, on sait déterminer en chaque point les valeurs de p et $\delta$, ce qui permet de déterminer la valeur de $K=p / \delta$ le long de l'interface, qu'il faudrait introduire dans le calcul par la méthode des coefficients de réaction pour retrouver les mêmes résultats que dans l'approche élastique rigoureuse (conduite en intégrant les déplacements par rapport à un point du massif très éloigné de l'ouvrage).

- Premier cas : problème bidimensionnel ; un écran vertical est mis en fiche dans le massif, et on examine les effets d'une charge horizontale exercée en tête, suivant le schéma de la figure 3 .

Il est intéressant de constater que les déplacements sont de même signe sur toute la hauteur de l'écran, ce qui n'empêche pas les pressions à l'interface de changer de signe entre le haut et le bas.

Pour respecter l'élasticité linéaire, il faut envisager une valeur de coefficient de réaction variable le long de l'écran, et il est indispensable de passer par des coefficients de réaction négatifs! Bien entendu, si l'objectif était de reproduire les seules pressions, d'autres solutions seraient envisageables, avec des coefficients variables, mais sans qu'il soit indispensable de passer par des valeurs négatives ; les déplacements seraient alors dénués de tout réalisme.

- Deuxième cas : problème bidimensionnel ; une bande de radier ou de dallage subit plusieurs chargements, suivant le schéma de la figure 4 .

Pour respecter l'élasticité linéaire, il faut ici aussi des coefficients de réaction variables le long de l'interface et négatifs dans certaines zones. Mais il faut remarquer que les coefficients $\propto$ réalistes $»$ dépendent non seulement du massif et de la structure, mais aussi du chargement. Ce constat est utile pour apprécier la validité des propositions de divers auteurs quant au choix des valeurs de coefficient de réaction à prendre en compte.

La méthode des coefficients de réaction, telle qu'elle est aujourd'hui mise en œuvre, n'a donc pas de capacité prédictive pour des éléments structurels surfaciques, sauf si elle est calée sur un constat expérimental portant sur un ouvrage suffisamment ressemblant ; c'est le contexte de la construction à l'identique, et le calcul n'est alors qu'une façade ou, au mieux, un moyen d'interpolation.

\section{5}

\section{Coefficients de poussée et de butée}

Nous avons vu que de tels coefficients sont introduits dans les codes de calcul appliquant la méthode des coefficients de réaction aux écrans de soutènement. Il est intéressant de remarquer que, si l'on prend en compte des coefficients de réaction très élevés, dans divers cas de figure le modèle coïncide avec le schéma de calcul par poussée-butée, ce qui est rassurant, et explique sans doute que le modèle ne conduise pas, dans des cas courants, à des déboires catastrophiques. Cette précaution s'avère malheureusement insuffisante dans des contextes présentant une certaine complexité. 

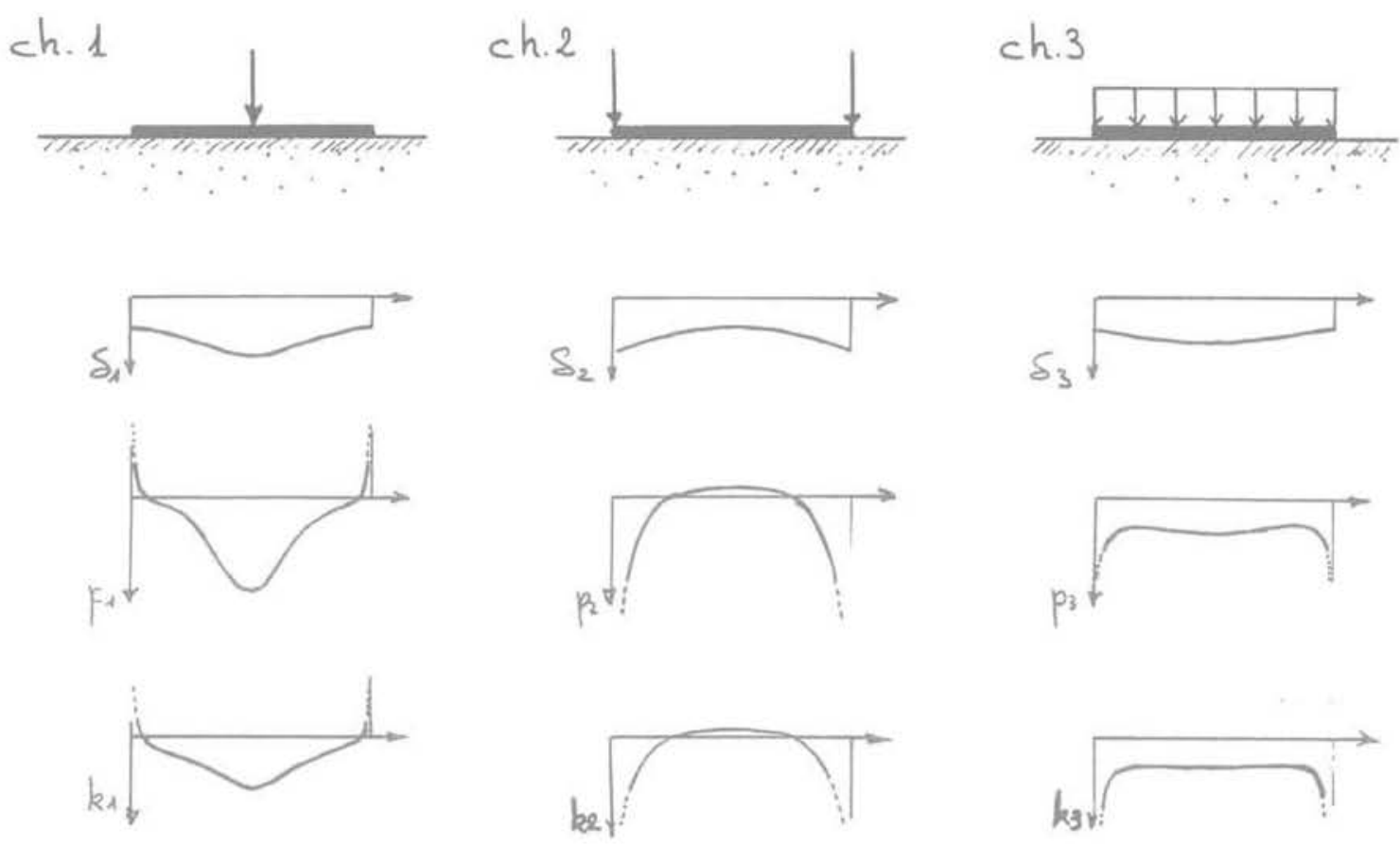

FIG.4 Massif semi-infini élastique, bande de dallage ou de radier, coefficient de réaction équivalent pour plusieurs chargements.

Semi infinite elastic footing, slab or raft strip, equivalent reaction coefficient for various loadings.
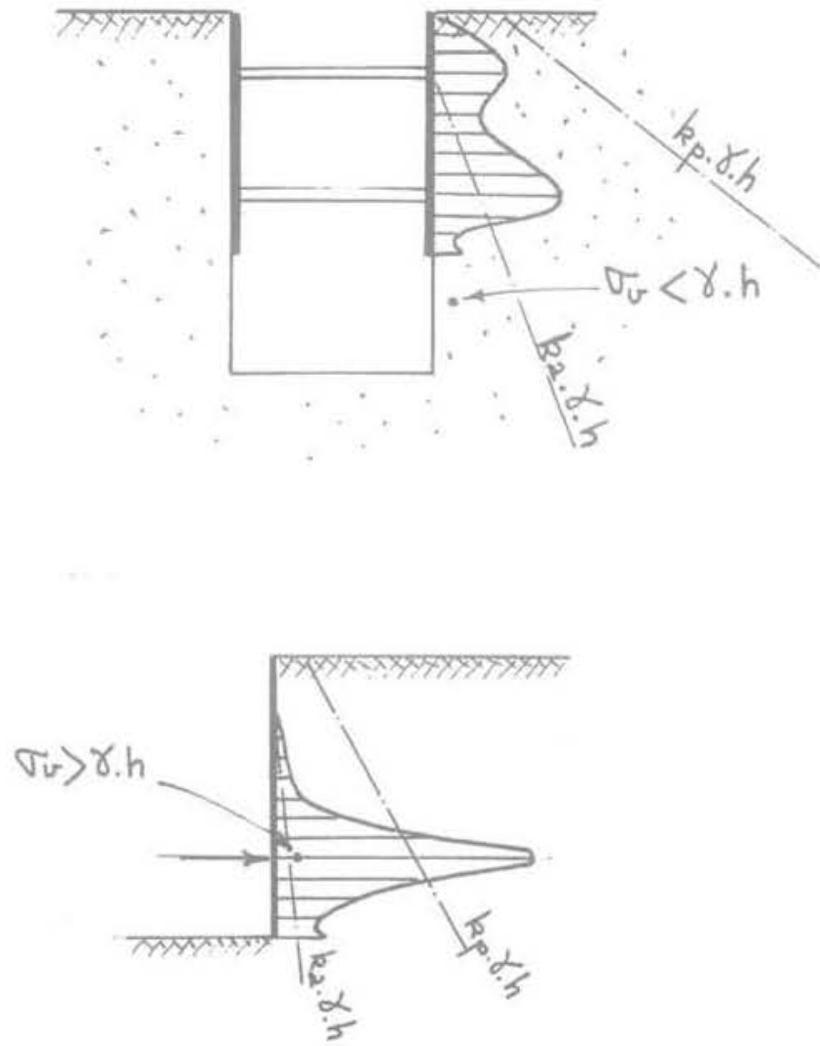

FG.S Validité des limitations de pression par les coefficients de poussée active ou passive.

Adequacy of limits on pressure by active or passive earth pressure coefficients.
Mais sil'on y regarde d'un peu plus près, ces limites traduisent une confusion regrettable entre $\sigma_{\text {, et }} \gamma . \mathrm{h}$. Physiquement, une limite de poussée ou de butée n'a de sens que si le sol est en état de plastification depuis une extrémité de l'interface jusqu'à la zone de contact considérée. Dans bien des configurations courantes, ces limites sont transgressées, voir exemples sur la figure 5.

\section{Symptômes inquiétants}

S'il existe sans aucun doute un domaine d'application du modèle des coefficients de réaction pour le dimensionnement de divers ouvrages simples, à l'inverse, on ne peut rien en attendre de bon dans d'autres cas. Ce qui est inquiétant est le constat que, même devant l'évidence de l'inadaptation du modèle pour tel ou tel problème, il reste la référence incontournable. Deux exemples :

- Dans le cas représenté sur la figure 6 se posait le problème de la stabilité et des efforts subis par la paroi dans l'hypothèse d'une fouille aval avec banquette le long d'une paroi moulée. Une modélisation par la méthode des éléments finis a été établie, tenant compte pour le sol du classique critère d'élastoplasticité à cinq paramètres $(c, \Phi, G, v, \psi)$ avec coupure de résistance en traction ; $v$ et $\psi$ étaient arbitraires car l'étude géotechnique ne les avait pas déterminés, mais leur influence sur les résultats était négligeable. Lorsque les résultats ont été présentés au maître d'œuvre, sa réponse a été : "Votre calcul est bien joli, mais il est indispensable d'en confirmer la validité par la méthode des coefficients de réaction., 


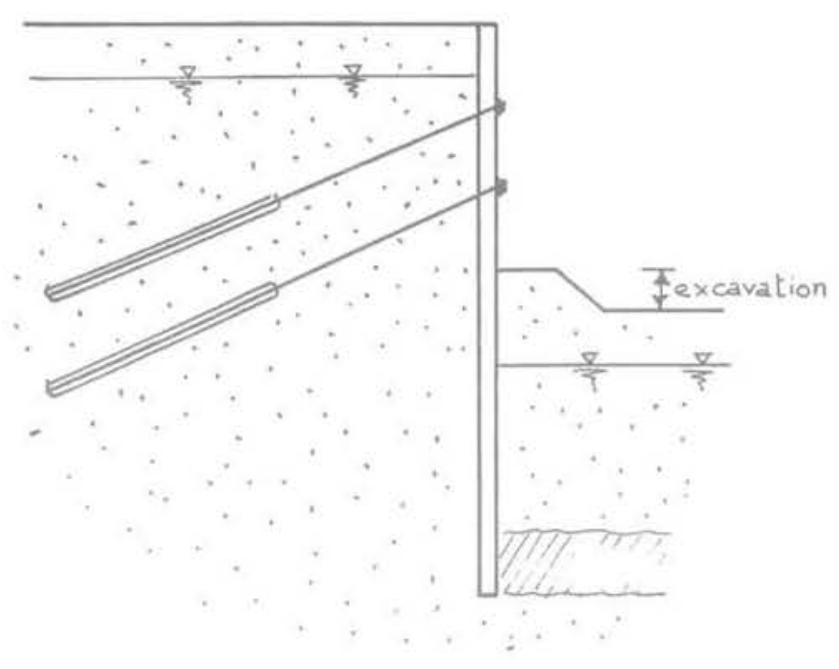

FIG.6 Écran de soutènement, objet de désaccords. Retaining wall, subject of contention.

- Le cas des ouvrages sous remblai est tout aussi édifiant (Fig. 7). Un ouvrage supportant une couverture de remblai importante, dimensionné en appliquant la méthode des coefficients de réaction, avait été l'objet d'une instrumentation assez poussée, motivée par l'utilisation de Pneusol pour maîtriser l'effet Marston d'amplification de $\sigma_{v^{\prime}}$ par rapport à $\gamma$.h. ; l'objectif était de s'assurer de la validité de la modélisation du Pneusol. Les constats expérimentaux ont confirmé la validité de la modélisation de l'effet du Pneusol, mais il a aussi été constaté que les efforts subis par la structure étaient très différents de ceux pris en compte pour le choix du ferraillage : les efforts de flexion restaient assez modestes pour que toutes les sections soient entièrement comprimées, alors que les calculs avaient conduit à un ferraillage important. Quels que soient les efforts consentis, il n'a pas été possible de reproduire avec la

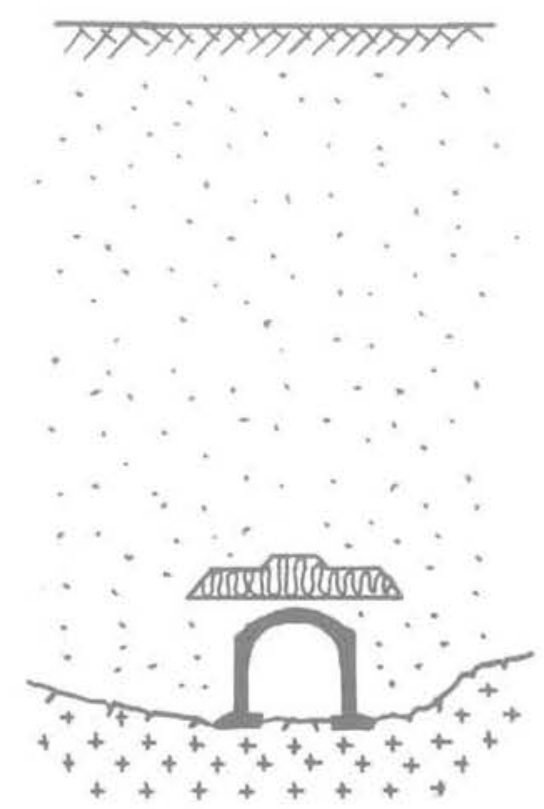

HG.7 Ouvrage sous remblai, objet de désaccords. Structure under backfill, subject of contention. méthode des coefficients de réaction les efforts et déplacement constatés. A l'inverse, la méthode des éléments finis a conduit à une coïncidence tout à fait satisfaisante avec les constats expérimentaux, efforts et déplacements.

Ajoutons ici que la méthode des coefficients de réaction ne conduit pas forcément à une appréciation sécuritaire des efforts, puisqu'elle ne permet pas de tenir compte des déformations de l'assise générées par la charge des remblais latéraux, déformations qui peuvent provoquer des efforts considérables dans le radier (se répercutant sur l'ensemble de la structure) (Fig. 8).

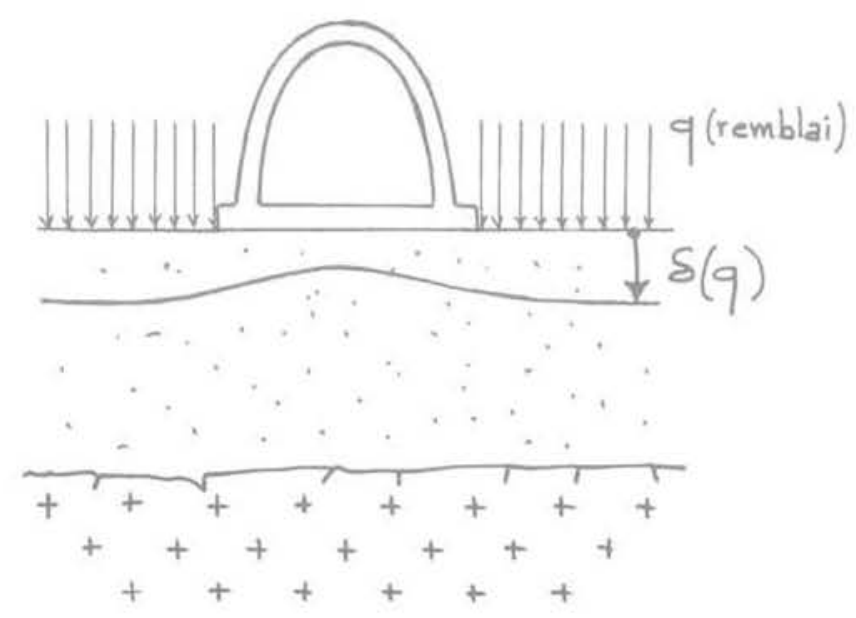

F1G.8 Effets des remblais latéraux sur le radier d'un ouvrage enterré.

Laterall backfill effect on the raft of an underground structure.

Mais ces constats ne furent pas suffisants pour ébranler la conviction du maitre d'cuvre, qui a systématiquement refusé, pour les ouvrages ultérieurs, tout calcul justificatif autre que basé sur la méthode des coefficients de réaction.

On est donc en présence d'une méthode de modélisation dénuée de tout réalisme, mais d'usage général pour traiter l'étude des interactions sol-structure, et qui bénéficie d'une confiance injustifiée. Tout se passe comme si les meilleurs spécialistes apportaient leur caution à son exploitation systématique. Si, en l'absence de toute solution alternative de modélisation, on peut admettre un silence gêné de la part des gens compétents, cette absence de mise en garde peut devenir de la complaisance lorsque des solutions alternatives fiables existent, et la publication de « raffinements $)$ est une circonstance aggravante : comment en vouloir au non spécialiste qui applique à tort et à travers une méthode dont personne ne conteste le bienfondé ?

\section{Domaine d'application}

Il semble qu'on peut utiliser le modèle des coefficients de réaction dans les cas suivants :

- éléments linéaires tels que les pieux, au moins pour des chargements simples ; 
- cas courants de soutènement dans un environnement simple, ou de radier, mais il reste à préciser quelles dispositions constructives doivent alors être respectées (par exemple, les tirants et les butons doivent vraisemblablement être traités de manières différentes s'ils sont ou non précontraints) ; il convient alors de déterminer si, dans certains cas, on peut se fier aux prédictions de déplacement.

En revanche, ce modèle devrait être exclu pour (liste non exhaustive de beaucoup) :

- la prévision des effets d'une excavation limitée par des écrans de soutènement sur le voisinage (sauf calage significatif sur un cas très voisin, mais alors on peut sans doute effectuer une comparaison directe) :

- les écrans de soutènement dès que les plates-formes amont et aval ne sont pas horizontales, ou avec écoulement ascendant d'eau souterraine dans la zone de butée (ajoutons qu'il ne suffit pas toujours de compléter l'étude par celle de la stabilité globale par un calcul à la rupture, et que les méthodes des éléments finis ou des différences finies sont parfois indispensables) ; - la justification des efforts subis par les ouvrages sous remblai reposant sur une assise qui ne soit pas très raide par rapport aux remblais.

\section{8}

\section{Conclusion}

La méthode des coefficients de réaction est aujourd'hui entre les mains de projeteurs non avertis de ses limites de représentativité. Les écrans de soutènement ne sont qu'un cas particulier de son application. Il est indispensable de mettre en garde les utilisa- teurs sur l'absence de réalisme que l'on peut attendre de ce modèle, dont l'utilisation devrait respecter un domaine d'application étroit.

Pour les projets importants et délicats, le recours à la MEF ou à la MDF avec des codes généraux s'impose.

Pour les projets courants, les efforts pourraient porter sur une simplification de l'utilisation de ces méthodes, simplification de génération du maillage et de définition du phasage constructif par exemple, et standardisation de la présentation des résultats, sans concession sur la finesse de discrétisation des problèmes. Les codes généraux permettent une étude représentative des états-limites de service (dans la mesure où les valeurs des paramètres de calcul sont représentatives...) comme des états-limites ultimes au sens de la Mécanique des Sols, alors on peut attendre un réel progrès de codes aux entrées-sorties simplifiées pour les cas courants.

On ne peut pas prétendre que les méthodes des éléments finis ou des différences finies conduisent à une modélisation irréprochable, même si les paramètres de comportement des sols sont représentatifs ; mais elles sont physiquement beaucoup plus satisfaisantes ; elles permettent une analyse globale du projet et de son environnement, qui a quelques raisons de refléter la réalité ; elles permettent d'apprécier les tendances résultant d'un aménagement des dispositions constructives (effet par exemple de la distance d'une paroi moulée quant aux sollicitations d'un ouvrage voisin existant). On peut surtout leur reprocher d'exiger que le projeteur consacre un peu plus de temps à un projet que s'il utilisait la méthode des coefficients de réaction ; mais il ne serait pas mauvais qu'un peu plus de matière grise soit consacrée au dimensionnement d'ouvrages dont un comportement déficient peut être lourd de conséquences désagréables. 